\title{
Metodología para la obtención del patrón de radiación y prueba de aislamiento en sistemas de comunicaciones vía satélite
}

\author{
A. Coronado-Rodríguez y F. Moumtadi \\ División de Ingeniería Eléctrica y $D$ epartamento de Telecomunicaciones \\ Facultad de Ingeniería, UN A M \\ E-mails: powerrun@servidor.unam.mx y fatimoum@yahoo.com.mx
}

(Recibido: noviembre de 2005; aceptado: junio de 2006)

\section{Resumen}

\begin{abstract}
El presente trabajo está enfocado en dos puntos muy importantes para la calidad en las comunicaciones vía satélite en la órbita geoestacionaria. La primera es el patrón de radiación de una antena con reflector parabólico, y la segunda, es el aislamiento en polarización cruzada. Ambos parámetros tienen una estrecha relación y proporcionan un dato muy importante respecto al desempeño del segmento terreno. Otro aspecto importante son las constantes revisiones y mejoras a las recomendaciones de dichos parámetros, a fin de reducir las interferencias entre redes de satélites y optimizar la órbita geoestacionaria.
\end{abstract}

Descriptores: Satélites, antenas, patrón de radiación, polarización cruzada, orbita geoestacionaria, estaciones terrenas.

\begin{abstract}
Thepres ent ar tic cleit is fo cused in two rel evant as pects in thegeo sta tion ary sat el litecom muni cations. The first is the par a bolic an tenna pat tern ra di a tion and the later is thecross-polarization isolation. Both pa ram eters havea closerela tion ship between them and they rep resent an im por tant as pect in the ground seg ment per for mance. Thereis other char acter isticin volved with an tenna pattern ra di a tion and iso la tion test, they aresub ject to frequent revi sion and im provements in or der to min i $m$ izethein ter fer. en ceprob lems between satel liteusersandthegeosta tion ary or bit opti mi za tion which means to get more or bital po si tions by reducing thean gu lar distancebetween sat el lites.
\end{abstract}

Keywords: Satel lite, an tenna, radiation dia gram crosspolar ization, geo-stationary or bit, earth stations.

\section{Antecedentes}

Las antenas están diseñadas, principalmente, para dar una distribución específica de energía en el espacio, a la vez que se minimizan las pérdidas y las reflexiones. Esa distribución es el patrón de radiación de la antena. La distribución incluye una región de alta concentración (ganancia) para iluminar un objetivo. Las radiaciones fuera de la dirección del haz principal deben ser severamente restringidas para evitar interferencias con otros sistemas o recibir interferencias desde otros. El nivel de los lóbulos laterales constituye el parámetro más delicado del diseño de antenas, otros parámetros de interés de la antena, son la polarización y profundidad de los nulos.

\section{Patrón de radiación}

El patrón de radiación de antena, el cual típicamente comprende un haz principal y una 
Metodología para la obtención del patrón de radiación y prueba de aislamiento ...

estructura de lóbulos laterales, es comúnmente representado como un trazo en dos dimensiones.

El ancho de haz marca su importancia en la medida de qué tan exacto debe apuntarse la antena y qué tan rápidamente la antena rechaza las señales fuera de la región deseada. El ancho angular del haz principal del patrón de radiación de la antena, es caracterizado por el ancho del haz a media potencia (HPBW), que se define como el ancho angular total entre los dos puntos que están $3 \mathrm{~dB}$ abajo del pico del haz principal. El HPBW es dependiente de la distribución de iluminación en la apertura de la antena y la dimensión de la apertura en el plano en el cual el patrón es medido.

Ganancia y directividad son cantidades que definen la capacidad de concentrar la energía de una antena en una dirección particular, y están directamente relacionadas al patrón de radiación de la antena. La ganancia $\mathrm{G}(\theta, \phi)$ de una antena en una dirección específica $(\theta, \phi)$ está definida como la razón de la potencia radiada por unidad de ángulo sólido $P(\theta, \phi)$, en la dirección $(\theta, \phi)$ a la potencia por unidad de ángulo sólido radiada desde una antena isotrópica, alimentada con la misma potencia total $\mathrm{P}_{\mathrm{T}}$. La antena isotrópica es una antena hipotética, la cual radia uniformemente en todas direcciones. Por lo tanto:

$$
G(q, f)=\frac{P(q, f)}{P_{T} / 4 p}
$$

Esta cantidad es una propiedad inherente de la antena e incluye pérdidas por disipación.

Cuando sólo nos importa qué tan bien se forma el haz en el espacio, en lugar de las pérdidas, entonces se aplica el término de directividad. La directividad $D(\theta, \phi)$ de una antena, no incluye las pérdidas por disipación y está definida como la razón de $\mathrm{P}(\theta, \phi)$ a la potencia por unidad de ángulo sólido, desde una antena isotrópica radiando la misma potencia $P_{R}$. Por lo tanto:

$$
D(q, f)=\frac{P(q, f)}{P_{R} / 4 p}
$$

La razón de $G(\theta, \phi)$ a $D(\theta, \phi)$ se conoce como "eficiencia de radiación" de la antena.

El valor de $G(\theta, \phi)$, donde la máxima radiación ocurre, se le conoce simplemente como "ganancia" de la antena, y en la mayoría de los casos se expresa en $\mathrm{dBi}$ (decibeles con relación a una antena isotrópica). Este valor corresponde al pico del haz principal del patrón de radiación, el cual es generalmente la dirección $(0,0)$ referida como "la dirección de apuntamiento o eje de apuntamiento de la antena".

El factor de mayor importancia para determinar la eficiencia de la utilización de la órbita geoestacionaria es el patrón de radiación asociado a la antena de la estación terrena, particularmente en la región angular de $0-50^{\circ}$ desde el eje de apuntamiento. Para cálculos de interferencia es conveniente describir la envolvente de los lóbulos laterales de la antena de una estación terrena de la siguiente forma:

$$
S(q)=A-B \log _{10} q d B i
$$

para $\theta>1^{\circ}$ que es el ángulo en grados desde el eje de apuntamiento, A y B son coeficientes numéricos representando el nivel de la envolvente a $1^{\circ}$, fuera de la línea de apuntamiento y la tasa de disminución de la envolvente, respectivamente.

El Comité Consultivo de Radiocomunicaciones de la UIT adoptó un patrón de radiación de referencia para usarse en cálculos de interferencia para antenas grandes con $A=32$ y $B=25$, conduciendo a una mínima separación entre satélites de $2.9^{\circ}$. Para reducir el espacio mínimo entre satélites a $2^{\circ}$ y por consecuencia, conseguir más posiciones orbitales, se creó una especificación más estricta $A=29$ y $B=25$, que está aplicándose actualmente en las antenas de estaciones terrenas en el modo de transmisión.

Otro factor importante en la calidad de una transmisión vía satélite es la polarización. En el 


\section{A. Coronado-Rodríguez y F. Moumtadi}

caso de una antena transmitiendo o recibiendo un campo linealmente polarizado, el componente cross-polarizado es el campo en el ángulo recto del componente co-polar. Por ejemplo, si el componente co-polar es vertical, el componente cross-polar será horizontal.

\section{Polarización cruzada y discriminación de la polarización}

La onda radiada por una antena consiste de un componente de campo eléctrico y un componente de campo magnético. Estos componentes son ortogonales y perpendiculares a la dirección de propagación de la onda, por lo que varían con la frecuencia de la onda. Por convención, la polarización de una onda electromagnética radiada es la curva trazada por el punto final del vector del campo eléctrico instantáneo, observada a lo largo de la dirección de propagación. La polarización puede ser clasificada como lineal circular 0 elíptica.

El método para obtener el re-uso de frecuencia, es transmitir dos señales en la misma banda de frecuencia (co-canal) y poniendo cada una en polarizaciones ortogonales; de este modo, se dobla la capacidad de información manejada por una satélite. Un requerimiento fundamental de la transmisión con polarización dual es mantener un buen nivel de aislamiento entre las dos polarizaciones, de tal manera que la interferencia co-canal sea mínima.

La antena es, por lo tanto, caracterizada para una polarización dada por un patrón de radiación para polarización nominal (co-polares) y un patrón de radiación para polarización ortogonal (polarización cruzada). La discriminación de polarización cruzada es generalmente máxima en el eje de la antena y se degrada para direcciones diferentes a aquella que corresponde a la dirección de máxima ganancia.

En un sistema de satélites con re-uso de frecuencia a través de polarización dual, un parámetro importante en la determinación de la calidad del sistema, es la razón de discriminación de polarización cruzada entre las señales co-polar y cross-polar, especialmente en la región del haz principal de la antena. A esto se le conoce como "interferencia cross-polar" (XPI). Un estándar obligado de pureza de polarización para las nuevas estaciones terrenas, ha sido impuesto por INTELSAT y menciona que el valor de XPI debe ser $30 \mathrm{~dB}$ para polarizaciones lineales. En el caso de EUTELSAT, que también usa polarización lineal en $11 / 14 \mathrm{GHz}$, el valor XPI es de al menos $35 \mathrm{~dB}$ en cualquier parte dentro del contorno de $-1 \mathrm{~dB}$ del haz principal.

Las Regulaciones de la UIT que nos incumben por ahora, son aquellas relacionadas con la interferencia con sistemas terrestres, en especial, el caso de una estación terrestre interfiriendo con un satélite en el apartado i que se refiere a las especificaciones para el diagrama de radiación de antenas de estaciones terrenas y su aislamiento en polarización cruzada donde se recomiendan los siguientes valores:

- El nivel de los lóbulos laterales debe ser de $\geq 12 \mathrm{~dB}$ con respecto al lóbulo principal.

- La diferencia entre lóbulos laterales no debe ser mayor a $2 \mathrm{~dB}$.

- $\quad$ El nivel de los nulos es de $4 \mathrm{~dB}$.

\section{Metodología para obtener el patrón de radiación}

Además de verificar la especificación, las pruebas de patrón de radiación sirven:

- Para calibración y almacenamiento de datos confiables.

- Como una inspección y procedimiento de control de calidad.

- Para evitar interferencias potenciales.

La metodología descrita aquí es fruto de la actividad y experiencia profesional de los auto res $y$ se divide en cinco partes:

1. Recopilación de datos. 
Metodología para la obtención del patrón de radiación y prueba de aislamiento ...

2. Configuración de la instrumentación.

3. Adquisición del trazo.

4. Evaluación del trazo.

5. Guardar el trazo y liberar el equipo.

\section{Recopilación de datos}

Esta parte está dedicada a recopilar toda la información necesaria para iniciar la prueba de patrón de radiación. Se necesitan los datos de la figura 1.

\section{Configuración de la instrumentación}

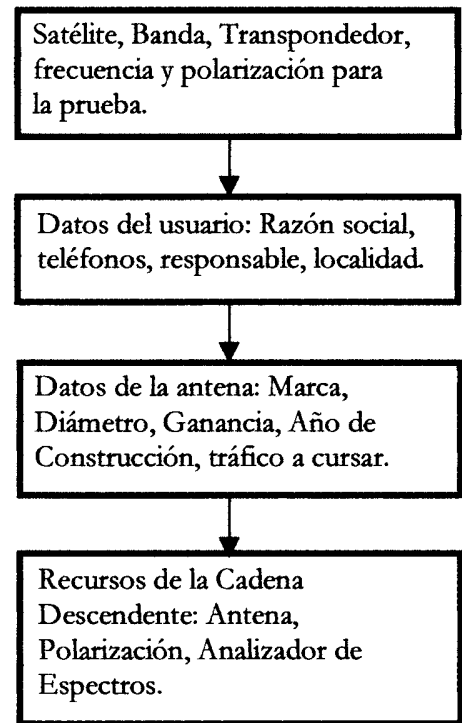

Figura 1. Recopilación de datos
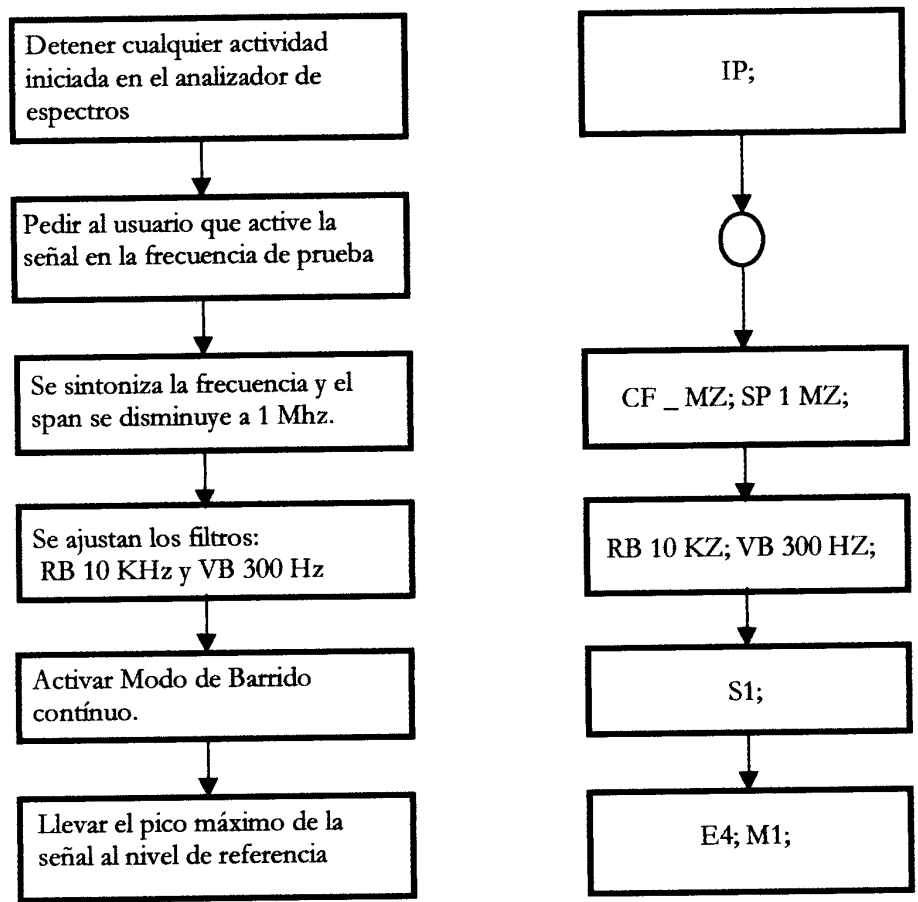

Figura 2. Configuración de la instrumentación 


\section{A. Coronado-Rodríguez y F. Moumtadi}
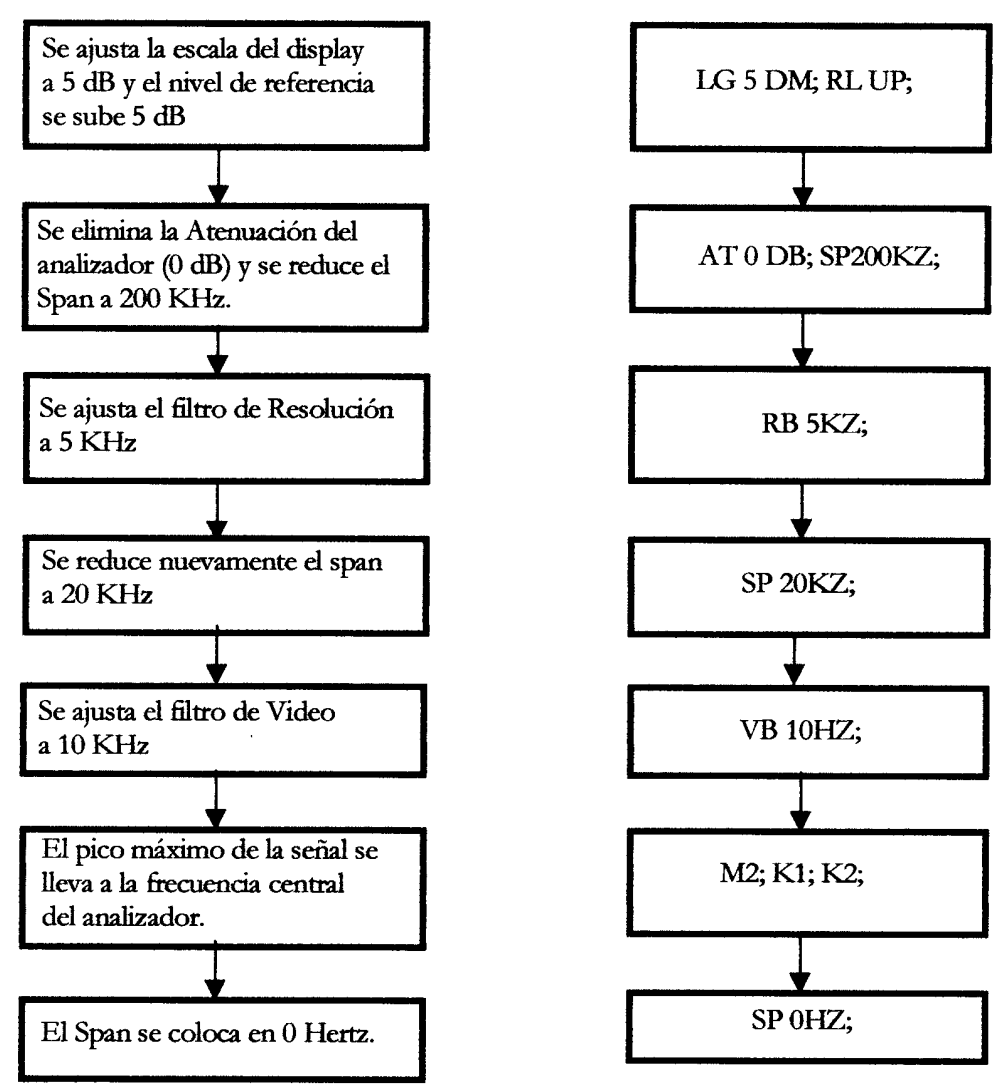

Figura 3. Configuración de la instrumentación (continuación)

Aquí se prepara y se configura la instrumentación que se va a utilizar a través de comandos enviados desde una red de computadoras a la instrumentación (Figura 2 y 3 ).

\section{Adquisición del trazo}

Esta parte de la metodología (Figura 4), consiste en:

- Borrar la marca.

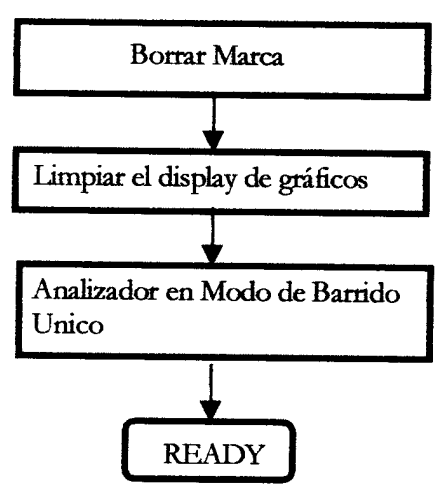

- Limpiar el trazo.

- Colocar al analizador en barrido único.

En este momento, todo está preparado para tomar una parte del trazo del patrón de radiación y el tiempo de barrido se ajusta al doble para tomar el trazo completo. También se puede usar la opción disparo de video del analizador para que el barrido se dispare cuando en la pantalla de video se

Figura 4. Adquisición del trazo 
Metodología para la obtención del patrón de radiación y prueba de aislamiento ...

detecte un aumento de nivel de la señal. Al usuario
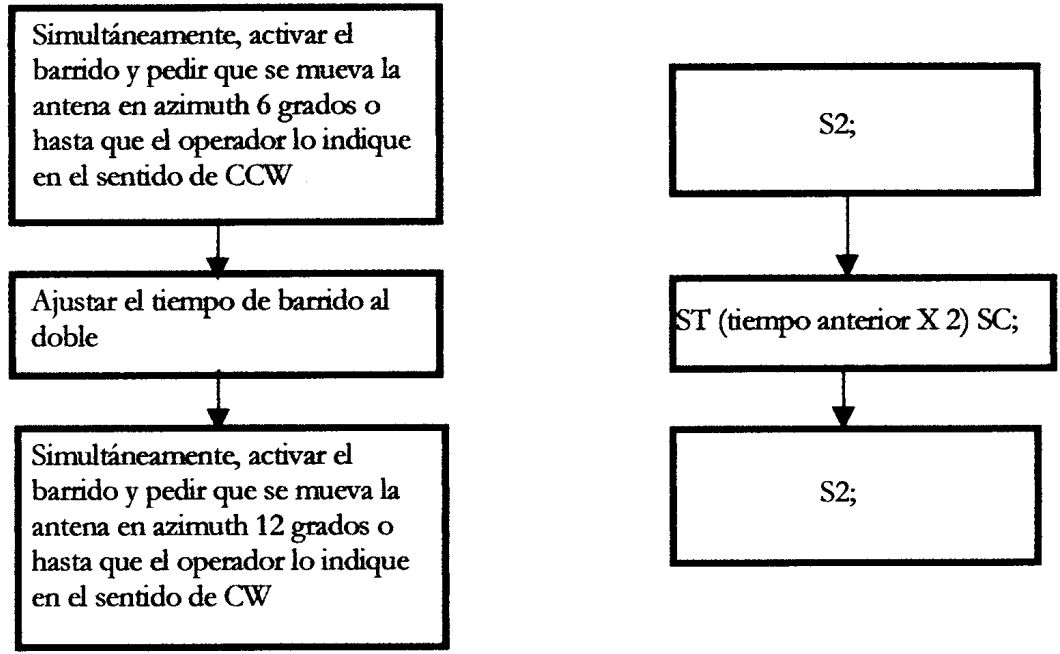

Figura 5. Adquisición del trazo (continuación)

se le pide que mueva la antena en forma constante, sin variar la velocidad, por lo que se detendrá hasta que el operador lo indique. Continuando:

Aquí, el trazo ya está en la pantalla y se procede a evaluarlo.

\section{Evaluación del trazo}

Con la ayuda de la marca del analizador se pueden visualizar en forma directa los niveles de los ló-

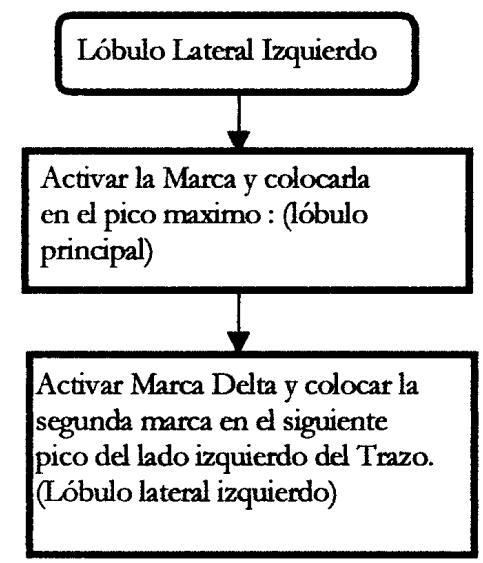

bulos laterales primarios del trazo del patrón de radiación, el primer lóbulo lateral izquierdo y el primer lóbulo lateral derecho (Figura 6 y 7).

\section{Guardar el trazo y liberar el equipo}

Esta parte de la metodología deja en condiciones iniciales el equipo utilizado:

- Guardar el trazo y sus parámetros principales como fecha, hora, satélite, banda, polarización, frecuencia, usuario, datos de la antena y nombre del operador.

Figura 6. Evaluación del trazo (lóbulo lateral izquierdo) 

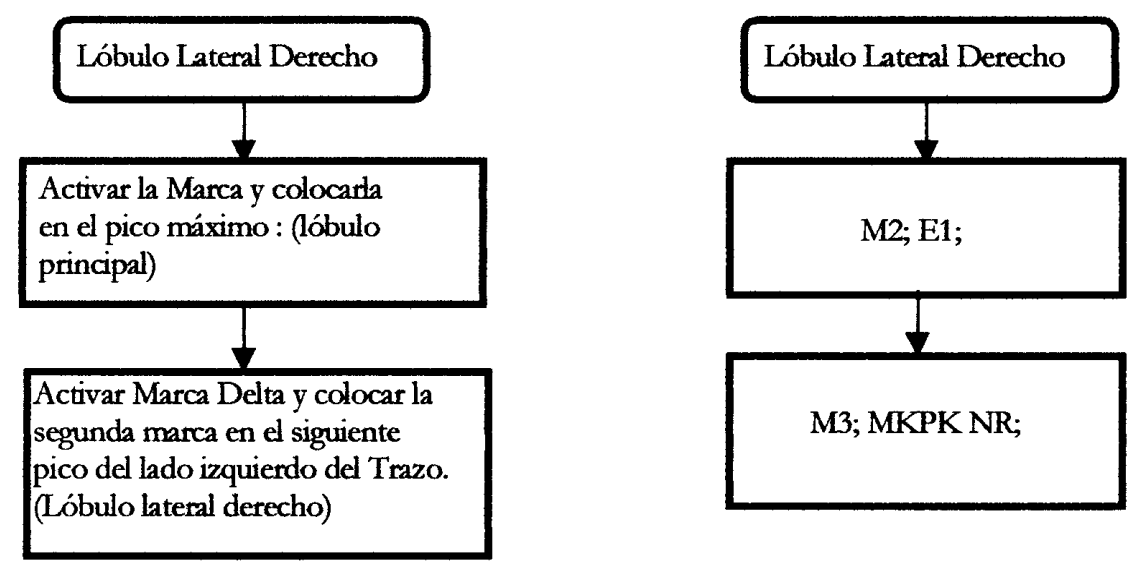

Figura 7. Evaluación del trazo (lóbulo lateral derecho)

- Enviar el comando de inicialización al analizador de espectros.

\section{Recomendaciones para la prueba de aislamiento de polarización}

Para el personal de pruebas:

1. Definir anchos de banda en cada transpondedor. Dichas ranuras deberán tener 300 $\mathrm{KHz}$ de ancho y libres en ambas polarizaciones, es decir, que tengan re-uso de frecuencia.

2. La antena con la que se medirá el aisamiento deberá encontrarse en condiciones de cambiar de una polarización a otra y tener una discriminación de polarización de 35 dB.

3. Los transpondedores del satélite deberán tener el mismo valor de atenuación.

4. La potencia de la señal es la potencia asignada en el cálculo de enlace más $5 \mathrm{~dB}$.

5. Seguir la norma del CCIR que menciona que el aislamiento debe ser de $32 \mathrm{~dB}$, como mínimo se acepta $28 \mathrm{~dB}$, dependiendo del tráfico a cursar.

Para el sistema de monitoreo de RF de portadoras:
1. Desplegar el trazo en tiempo real en una sola pantalla en ambas polarizaciones.

2. Habilitar las marcas y sus funciones como Marca Delta del analizador de espectros en la pantalla de gráficos del sistema, para tener una lectura precisa del valor del aislamiento

\section{Para los usuarios que desean ajustar el aislamiento en su antena:}

1. Tener la posibilidad de enviar portadora limpia (CW).

2. Ajustar su equipo en la ranura más cercana a su frecuencia de operación.

3. Ajustar la potencia y subir la señal en prueba.

4. Antes de mover el polarizador, hacer una marca que nos sirva de referencia para saber en qué lugar estaba el polarizador antes de iniciar la prueba.

5. Tener las herramientas necesarias o facilidades para rotar el polarizador sin apagar la señal y bloqueando, lo menos posible, el área de apertura del reflector.

6. En caso de no lograr el valor adecuado se recomienda: 
Metodología para la obtención del patrón de radiación y prueba de aislamiento ...

a) Hacer un apuntamiento fino de la antena con la ayuda del centro de monitoreo.

b) Asegurarse de rotar adecuadamente el alimentador.

c) Efectuar la prueba en condiciones de cielo despejado.

d) Asegurarse que el alimentador está en el foco y que está totalmente fijo.

e) No reemplazar partes de la antena, reflector ni equipo asociado.

7. Al finalizar exitosamente la prueba se debe desactivar la portadora.

8. Medir la portadora con sus parámetros asignados y comisionar.

\section{Conclusiones}

La metodología descrita es fruto de la práctica profesional y del trabajo en campo, su aplicación es importante para la puesta en marcha de una estación terrena y la calidad de transmisiones en un enlace vía satélite. Esta metodología y reco- mendaciones están trabajando actualmente en cuatro sistemas de monitoreo de diferentes proveedores; por lo que se puede concluir lo siguiente:

- La metodología y recomendaciones descritas son de gran valor para el adecuado desempeño de un enlace satelital en un ambiente tan congestionado de tráfico.

- Estos mismos procedimientos y recomendaciones, pueden ser tomados como guía por los usuarios del Sistema de Satélites Mexicanos, como parte de sus rutinas de mantenimiento en sitio, y así garantizar la calidad de sus enlaces vía satélite.

- La información para la elaboración del presente artículo es escaso, por tanto, lo escrito aquí es fruto de la experiencia con cuatro sistemas de monitoreo diferentes.

- El procedimiento para obtener el patrón de radiación y las recomendaciones para el aislamiento de polarización sirvieron para eliminar los problemas de interferencia ocasionados entre satélites canadienses y mexicanos.

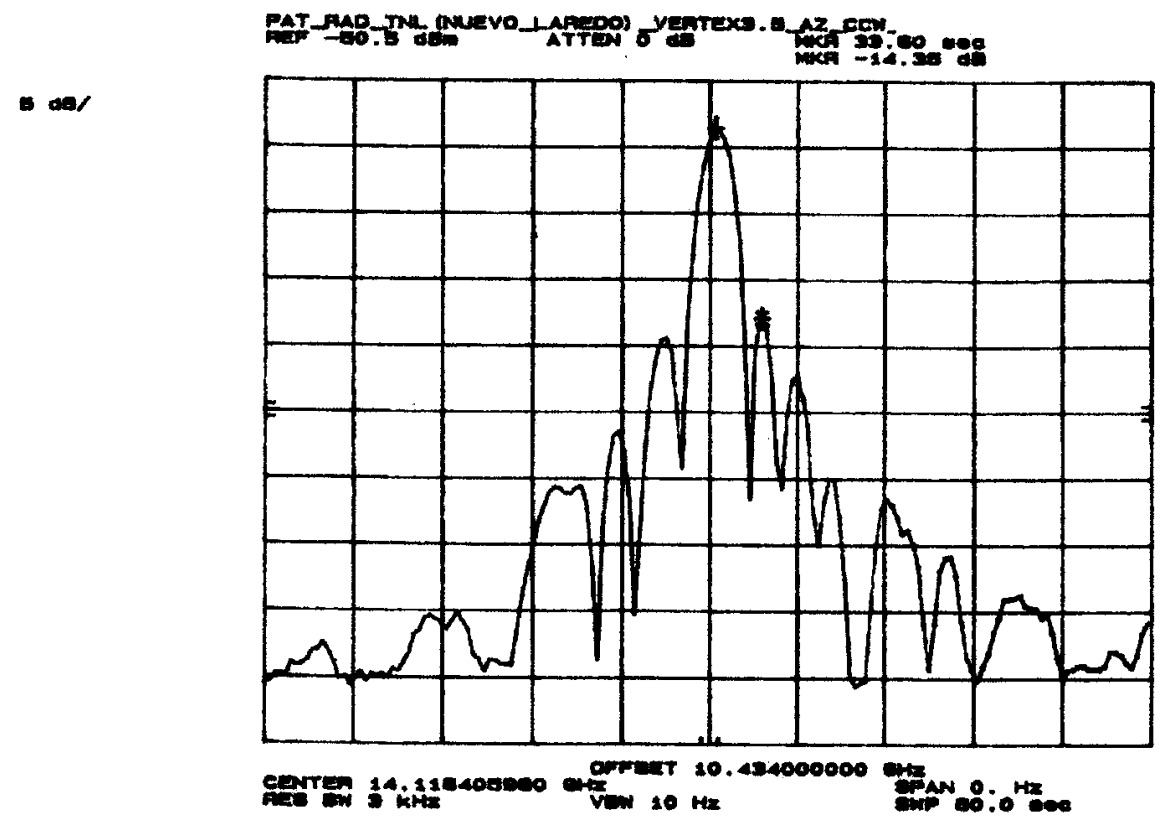

Figura 8. Patrón de radiación mostrando el nivel del lóbulo lateral derecho 
A. Coronado-Rodríguez y F. Moumtadi

- La elaboración de los procedimientos y recomendaciones se obtuvieron con base en la experiencia profesional y análisis de problemas en campo.

La figura 8 muestra un Patrón de Radiación obtenido con el anterior programa.

\section{Referencias}

UIT. (2004). Recomendaciones 465 y 580 del CCIR, Volumen IV, Parte 1 y Volumen IX, Parte 2, Servicio Fijo por Satélite.

Coronado-Rodríguez A. (2003). Propuestas de mejoras al sistema de monitoreo de satélites de Eutelsat. Tesis de Maestría en Ingeniería. Universidad Nacional Autónoma de México, Ciudad Universitaria, México.

HP Company (1995). HP Spectrum Analyzer 0 per ating and Programming $M$ anual .

\section{Semblanza de los autores}

Alejandro Coronado- Rodríguez. Obtuvo los títulos de ingeniero en computación y maestría en ingeniería eléctrica por parte de Ia UNAM. Actualmente es consultor experto en satélites de la ITU y profesor de asignatura en la Facultad de Ingeniería de la UNAM.

Fatima M oumtadi. Obtuvo los grados de maestría y doctorado en el 2002 en la Facultad de Radiodifusión y Televisión en la Universidad de Telecomunicaciones e Informática de Moscú. Se desarrolla profesionalmente en el área de radiofrecuencia. Actualmente es profesora de asignatura en la Facultad de Ingeniería de la UNAM 